Presented at: Accelerator Based Sources of

Infrared and Applications

San Diego, CA

July 29-30, 1997

BNL- 64881

\title{
Infrared Microspectroscopy with Synchrotron Radiation
}

\author{
G. L. Carr and G. P. Williams
}

National Synchrotron Light Source

Brookhaven National Laboratory

Upton, NY 11973-5000

September 1997

\footnotetext{
National Synchrotron Light Source

Brookhaven National Laboratory

Upton, NY 11973
}

Work performed under the auspices of the U.S. Department of Energy, under contract DE-AC02-76CH00016 


\section{DISCLAIMER}

This report was prepared as an account of work sponsored by an agency of the United States Government. Neither the United States Government nor any agency thereof, nor any of their employees, makes any warranty, express or implied, or assumes any legal liability or responsibility for the accuracy, completeness, or usefulness of any information, apparatus, product, or process disclosed, or represents that its use would not infringe privately owned rights. Reference herein to any specific commercial product, process, or service by trade name, trademark, manufacturer, or otherwise does not necessarily constitute or imply its endorsement, recommendation, or favoring by the United States Government or any agency thereof. The views and opinions of authors expressed herein do not necessarily state or reflect those of the United States Government or any agency thereof. 


\section{DISCLAMIER}

Portions of this doenement may be illegible in electronic image produets. Images are produced from the bet available original document. 


\title{
Infrared Microspectroscopy with Synchrotron Radiation
}

\author{
G.L. Carr and G.P. Williams \\ National Synchrotron Light Source \\ Brookhaven National Laboratory, Upton, NY 11973 \\ AECEIVED \\ DEC 034997 \\ Abstract \\ OSTI
}

Infrared microspectroscopy with a high brightness synchrotron source can achieve a spatial resolution approaching the diffraction limit. However, in order to realize this intrinsic source brightness at the specimen location, some care must be taken in designing the optical system. Also, when operating in diffraction limited conditions, the effective spatial resolution is no longer controlled by the apertures typically used for a conventional (geometrically defined) measurement. Instead, the spatial resolution depends on the wavelength of light and the effective apertures of the microscope's Schwarzchild objectives. We have modeled the optical system from the synchrotron source up to the sample location and determined the diffraction-limited spatial distribution of light. Effects due to the dependence of the synchrotron source's numerical aperture on wavelength, as well as the difference between transmission and reflection measurement modes, are also addressed. Lastly, we examine the benefits (when using a high brightness source) of an extrinsic germanium photoconductive detector with cone optics as a replacement for the standard MCT detector.

Keywords: Schwarzchild objective, diffraction, infrared detector, infrared beamline.

\section{Introduction}

Infrared microspectroscopy is a technique where the performance is usually limited by the source brightness. In a typical microspectroscopy measurement, apertures are placed at field stops in order to confine the infrared to a particular region of interest in a specimen. The infrared flux that reaches the detector, and therefore the signal-tonoise, decreases with aperture size, and for a conventional (thermal) infrared source one reaches a practical limit for spatial resolution of about $25 \mu \mathrm{m}$. This size scale is larger than the diffraction-limit, and the spatial resolution in this instance is determined by geometrical considerations, independent of the wavelength or measurement mode. The synchrotron infrared source is much brighter than the thermal source ${ }^{1}$, and good signal-to-noise can be achieved with apertures that are effectively at, or below, the diffraction limit. In this situation, the available spatial resolution depends on the wavelength as well as the specific apertures of the optical system.

Previously we reported the performance of an infrared microspectrometer system installed at beamport U2B of the NSLS ${ }^{2,3}$. A substantial amount of the synchrotron's intrinsic brightness was lost due to the small extraction aperture of this port, and a simple optical design for interfacing the infrared light to a microspectrometer was adequate for achieving optimal performance. Despite the less-than-ideal performance, a substantial advantage was realized over the microspectrometer's conventional thermal source, and the practical limit for spatial resolution was often determined by diffraction ${ }^{4}$. Several new infrared beamlines with large apertures are presently under construction at the NSLS. Since these should be capable of delivering the intrinsic synchrotron brightness, careful attention to the microscope's optical interface (and the microscope itself) is necessary to ensure that the greater available source brightness is not lost due to the optical system.

In this paper, we report analysis results of both the infrared beamline optical system as well as the microspectrometer up to the location of the specimen. This analysis indicates that, in many situations, spherical mirrors can be used without incurring a substantial loss in the synchrotron's intrinsic brightness. The analysis also shows how the diffraction-limited spotsize at the sample varies with the measurement conditions. 


\section{Synchrotron Source and Beamline Optics}

Synchrotron radiation is emitted from relativistic electrons as they are accelerated along a circular trajectory. Though the geometry of the total source is somewhat complex ${ }^{1}$, the portion of the source that produces the brightest radiation is defined by the electron beam cross section, which is a few hundred microns in size (or less) for most synchiriotron radiation facilities. The angle into which the synchrotron radiation is emitted depends on frequency according to $\theta_{v}=1.6(\mathrm{p} v)^{-1 / 3}$, where $\rho$ is the electron bend radius (in $\mathrm{cm}$ ) and $v$ is the frequency of light (in $\mathrm{cm}^{-1}$ ). A typical value for mid.infrared (e.g. $2000 \mathrm{~cm}^{-1}$ or $\lambda \sim 5 \mu \mathrm{m}$ ) light is about 20 milliradians (mr). Immediately one can see that the effective source size, determined by diffraction, is $250 \mu \mathrm{m}$; comparable to the electron beam cross section. Therefore, the brightest portion of the source appears as a diffraction-limited point source, and to maintain that brightness, the beamline and microscope optical systems must also deliver diffraction-limited performance.

The NSLS infrared beamlines intended for microspectroscopy are designed around an extraction system that collects $\sim 40 \mathrm{mr}$ from the VUV ring $(\rho=191 \mathrm{~cm})$. Per horizontal milliradian, this opening angle collects essentially $100 \%$ of the infrared down to $250 \mathrm{~cm}^{-1}$, and the collection efficiency decreases slowly with frequency below this value. The extraction system is therefore more than adequate for the majority of infrared microspectroscopy measurements, which are performed at frequencies above $400 \mathrm{~cm}^{-1}$.

Beam extraction is accomplished with a combination of plane and ellipsoidal mirrors which collect the infrared and focus it through a diamond window. This window separates the storage ring vacuum from the remaining optical components. A diagram of a typical infrared extraction system (but without the vacuum components) is shown in Figure 1. Assuming that the ellipsoidal mirror is optimally positioned, the focus becomes an ideal, nearly $1: 1$ reproduction of the original source, and serves as a new source point for the remaining optical systems. The infrared light from the diamond window focus is collimated and relayed to the interferometer of the IR microspectrometer. We have the option of using off-axis paraboloids or spherical mirrors for collimation, and the performance for each type will be addressed shortly.

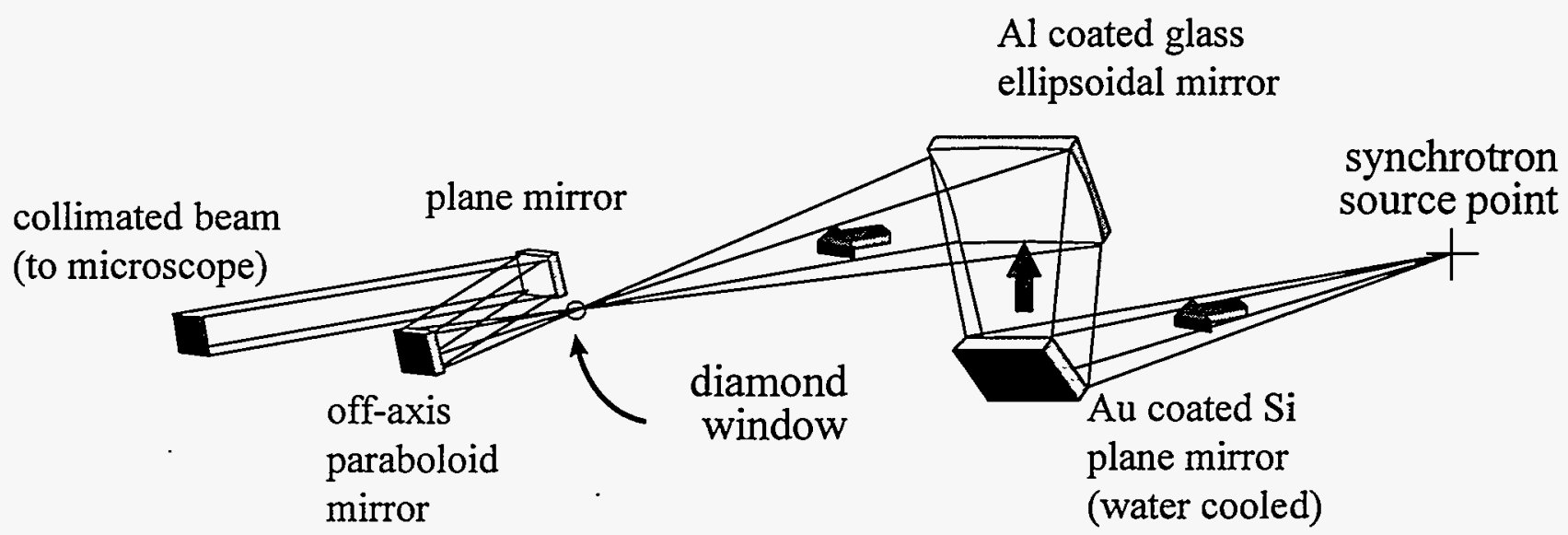

Figure 1. Schematic of synchrotron infrared beamline extraction optics. All components before the diamond window are at ultrahigh vacuum (UHV).

\section{Microscope optics}

The microspectrometer system in use at the NSLS is a Spectra-Tech Ir $\mu s^{\mathrm{TM}}$, provided by the Northrop Grumman Corp. as part of a collaborative research program. It consists of a standard Fourier Transform InfraRed (FTIR) spectrometer for the mid-infrared $\left(400 \mathrm{~cm}^{-1}\right.$ to $\left.\sim 4000 \mathrm{~cm}^{-1}\right)$, followed by a confocal microscope system and IR detector. A schematic of the instrument is shown in Figure 2. Beginning with the FTIR spectrometer, the collimated infrared is modulated by the moving mirror of the interferometer and transferred to the microscope system. The 
microscope is based on two Schwarzchild reflecting elements, with the specimen to be investigated placed at their common focus. This confocal arrangement reduces difffraction effects while enhancing contrast. Typically, the illuminating element that precedes the sample is called the "objective" whereas the element following the sample is the "condenser". This agrees with standard microscope nomenclature when the instrument is used as an optical microscope to view specimens, but is reversed from the standpoint of the infrared. Indeed, the objective and condenser become one and the same when the instrument is used in reflection mode. Schwarzchild objectives are free from on-axis aberrations while providing a large working distance. The objectives and condenser are used at finite conjugates in the same manner as standard microscope objectives. Adjustable apertures can be placed at the focal planes before the objective and after the condenser to serve as field stops, and are referred to as the "upper aperture" and "lower aperture" respectively.

In a transmission measurement, the upper aperture serves to define the sample area that is illuminated with infrared radiation from the objective. The lower aperture, in conjunction with the condenser, restricts the field of view that the detector "sees". The collimated infrared from the FTIR spectrometer is focused with a mirror onto the upper aperture location, and a similar mirror collects the light from the lower aperture and transfers it to the detector.

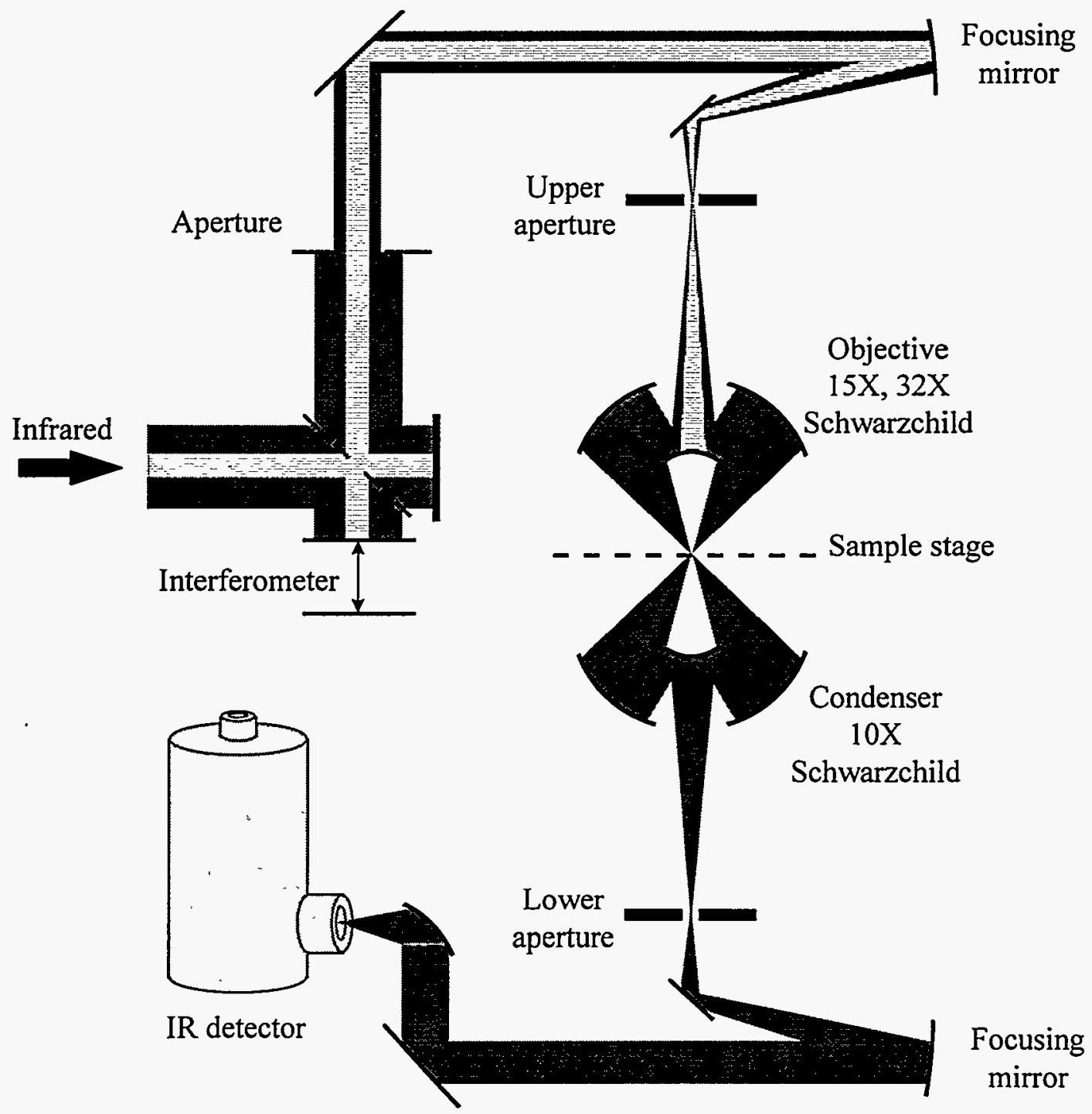

Figure 2. Schematic diagram of an infrared microspectrometer system such as the Spectra Tech Ir $\mu$ s. 


\section{Optical Analysis}

As mentioned above, a primary concern with the synchrotron source is maintaining its high brightness through the entire optical system, i.e. that the optical system be diffraction-limited. For this reason, we have modeled most of the beamline and microscope optical systems using Zemax optical design software ${ }^{5}$. We consider the microscope optics first, and then return to the beamline optics.

The Ir $\mu$ s microspectrometer can be used with a number of objectives for focusing light onto a specimen. The standard objectives have magnifications of $15 X$ (N.A. $=0.57$ ) and $32 X$ (N.A. $=0.65$ ). A fixed $10 X(N . A .=0.68)$ condenser collects the infrared in a transmission measurement. Schwarzchild objectives can perform at the diffraction limit over a field of view that easily exceeds the area illuminated by the synchrotron source. Therefore, the objectives should not cause any degradation to the synchrotron's intrinsic brightness, and our analysis confirms this.

Collimated light from the interferometer is focused onto the upper aperture by a spherical mirror, which for the Ir $\mu \mathrm{s}$ is used slightly off-axis. When used at its full aperture $(\sim \mathrm{f} / 13)$ in conjunction with the $15 \mathrm{X}$ objective, this element is not diffraction-limited and would lead to a $\sim 4$-fold loss in the synchrotron's brightness at the shorter wavelengths. Also, the positioning of this optic is critical for minimizing aberrations. These aberrations decrease rapidly with aperture, and the performance is close to the diffraction limit for the $32 \mathrm{X}$ objective which accepts light from the upper aperture at effectively $\mathrm{f} / 25$. This smaller aperture is illustrated in Figure 2 by the light gray hatched region which represents the beam cross section accepted by the $32 \mathrm{X}$ objective (as compared to the $15 \mathrm{X}$ ). A similar mirror system collects light from the lower aperture focus and transfers it to the detector system. The performance of this latter mirror system is less critical since the detector is designed to receive infrared from sample regions much larger than the diffraction limit, and we do not treat it here. However, in situations where the detector area must be optimized for small areas, or when replacing the single element detector with an imaging focal plane array, this spherical mirror (and any other focusing elements between the condenser and detector) will need consideration.

Modeling of the beamline optics from the source up to the diamond window focus confirms that no appreciable aberrations are introduced. The same result is obtained when an off-axis paraboloid is used to collimate the infrared before introducing into the Ir $\mu$ S FTIR system. We also consider the effects of a spherical mirror at this location since the next element is the spherical mirror that focuses the light onto the upper aperture location and there is an opportunity to compensate for some of its aberrations. This turns out to be the case when the $32 \mathrm{X}$ objective is in use (to the point that aberrations are completely negligible) as identical spheres can be used to match the NSLS VUV ring's $40 \mathrm{mr}(\mathrm{f} / 25)$ to this objective.

We can illustrate the various aberration effects caused by various combinations of collimating and upper aperture focusing mirrors by calculating the point spread function (PSF) at the sample location for the wavelengths of interest. The calculation begins at the synchrotron source point and all optics (except for the FTIR beamsplitter) are included up to the specimen location beneath the Schwarzchild objective. The results are shown graphically in Figure 3 for a $15 \mathrm{X}$ objective. We refer to the beamline collimating mirror as " $\mathrm{A}$ " and the upper aperture focusing mirror as " $\mathrm{B}$ ", and consider three scenarios for these mirrors at a wavelength of $3 \mu \mathrm{m}$. The upper right panel is for the first case of an ideal (diffraction-limited) system where both A and B are off-axis paraboloids, resulting in the standard Airy pattern. The same pattern is produced with spherical mirrors if used at normal incidence. The upper left panel is for case 2 where both $A$ and $B$ are spherical mirrors, used slightly off-axis. The off-axis angle for $A$ is kept minimal, while the angle for $B$ is fixed by the microscope to $\sim 7^{\circ}$ angle of incidence. The pattern is much larger and complex, and clearly demonstrates the effect of using spherical mirrors off-axis. Replacing mirror $A$ with an ideal off-axis paraboloid (case 3, lower left panel) yields only a slight increase in performance. Near optimal performance is achieved at $\lambda=10 \mu \mathrm{m}$ for even the worst case (lower right panel) since the diffracted spotsize is much larger. Note that in all cases it was assumed that the focusing mirror for the upper aperture is optimally positioned, and the $15 \mathrm{X}$ objective uses mirror $B$ at $\mathrm{f} / 13$. We have not determined the precise positioning tolerance for the upper aperture spherical mirror.

The calculated PSF for a $32 \mathrm{X}$ objective with matched off-axis spherical mirrors confirms that this optical system is capable of diffraction-limited performance across the entire spectral range of interest (see upper left panel of Figure 5). The improved performance (over the $15 \mathrm{X}$ ) stems primarily from the smaller acceptance of the $32 \mathrm{X}$, which utilizes 
mirror $B$ at the much smaller aperture of $f / 25$. Thus, with the exception of the $15 X$ objective at short wavelengths, the intrinsic source brightness can be maintained through to the specimen using spherical mirror optics.

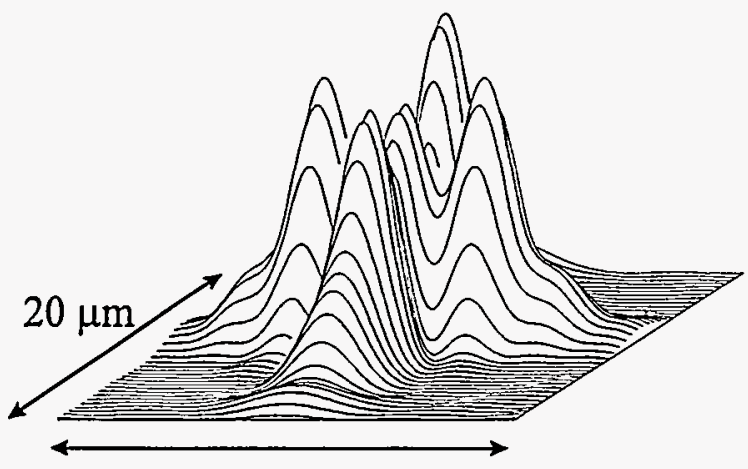

$20 \mu \mathrm{m}$

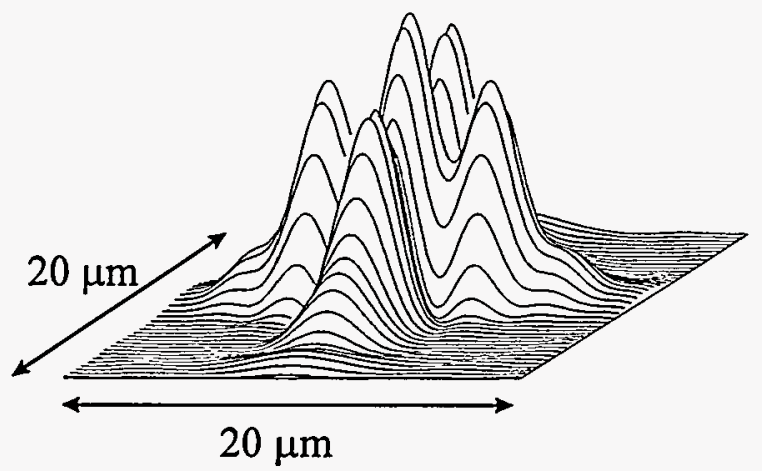

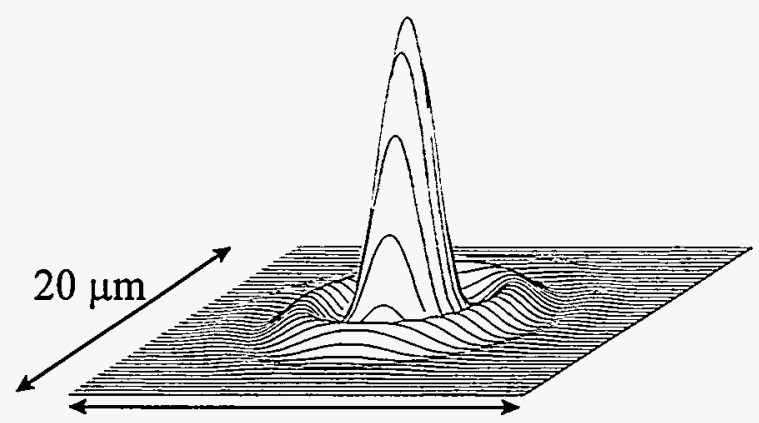

$20 \mu \mathrm{m}$

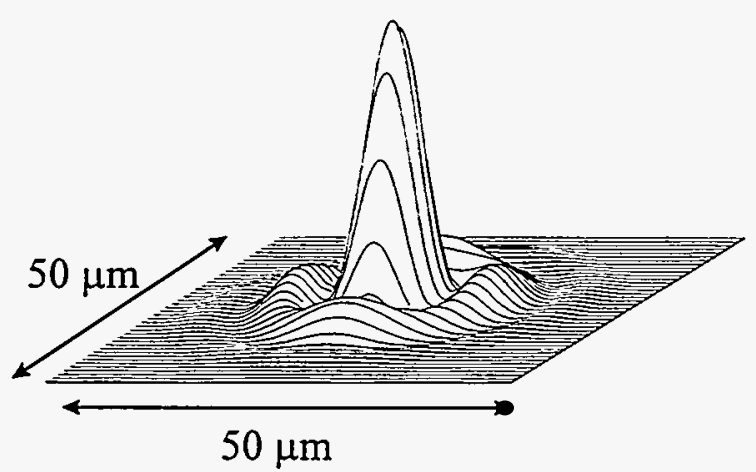

Figure 3. Point spread functions at the specimen location of the microspectrometer with a $15 \mathrm{X}$ objective. Calculations for different combinations of the beamline collimating mirror $(A)$ and upper aperture focusing mirror (B) preceding the microscope's objective. Counter-clockwise from upper right: 1) A \& B both ideal paraboloids (or spheres on-axis), $\lambda=3 \mu \mathrm{m}$; 2) A \& B both off-axis spheres, $\lambda=3 \mu \mathrm{m} ; 3$ ) $A=$ ideal paraboloid, $B=$ off-axis sphere, $\lambda=3 \mu \mathrm{m}$; 4) A \& B both off-axis spheres, $\lambda=10 \mu \mathrm{m}$.

Having delivered the optimum synchrotron brightness into the microscope, it becomes practical to set the microscope's upper aperture to a very small size such that it appears as a point source for the objective. The illuminated spotsize at the specimen is then determined by diffraction and consequently varies with wavelength.

Another wavelength-dependent effect comes into play with the synchrotron source. As noted previously, the vertical angle into which synchrotron radiation is emitted varies with frequency. For the NSLS VUV ring with a bending radius of $191 \mathrm{~cm}$, one finds that infrared at $3000 \mathrm{~cm}^{-1}$ is emitted into $19 \mathrm{mr}$ while $400 \mathrm{~cm}^{-1}$ light is emitted into $37 \mathrm{mr}$ (see Figure 4). Therefore, matching the optical system at $400 \mathrm{~cm}^{-1}$ results in $3000 \mathrm{~cm}^{-1}$ infrared filling only $1 / 2$ of the vertical aperture of the microscope optics. Variations in the filling of the entrance aperture of a Schwarzchild objective affect the diffracted spotsize at the sample location. We illustrate this in Figure 5, which shows the point spread function at a fixed wavelength of $3 \mu \mathrm{m}$ wavelength for three illumination conditions of the objective's entrance pupil. We have kept the wavelength fixed so that only the affects of vertical filling affect the point spread function. The upper left shows the fully illuminated pattern, which yields basically the same pattern (and therefore resolution) along both transverse directions. We again use the complete optical system for the calculation, including the off-axis spherical mirror upstream of the Schwarzchild objective (32X), leading to the lack of perfect circular symmetry in the 


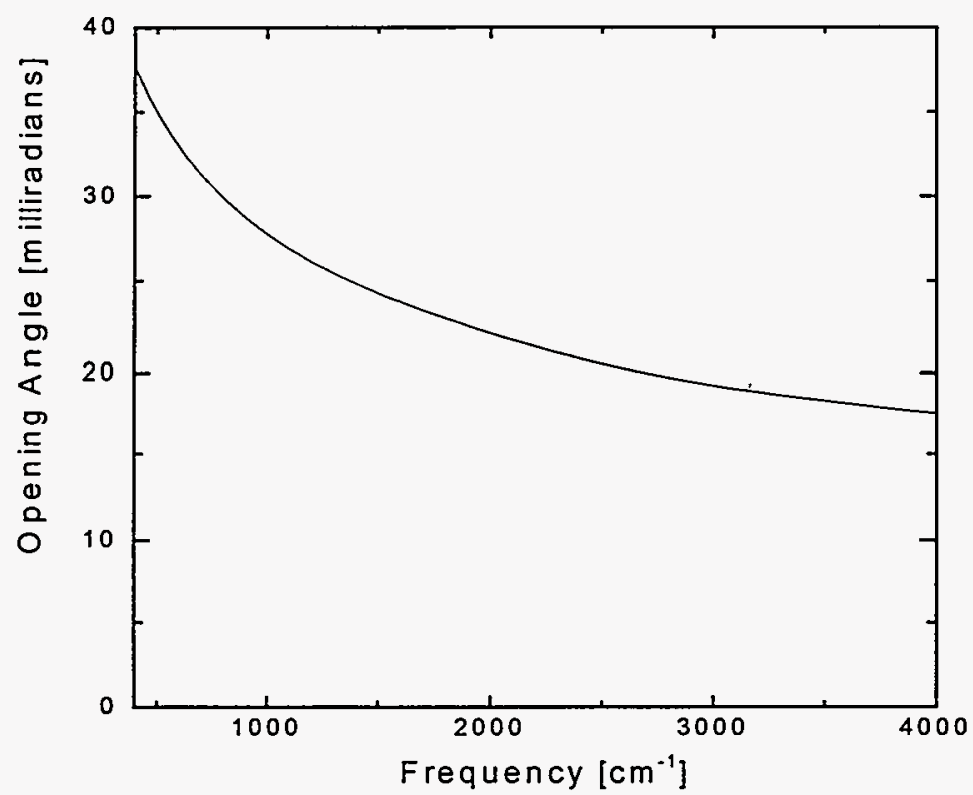

Figure 4. Intrinsic opening angle for synchrotron radiation from the NSLS VUV ring as a function of infrared frequency. pattern. The middle portion of the figure shows the effect of partial filling in the vertical direction; leading to a broadening of the diffraction pattern in the same direction while enhancing the strength of the secondary fringes in the horizontal direction. The lower right shows the effect of filling somewhat less than half the vertical entrance pupil such that the illuminated region is divided into two nearly isolated regions. This causes the pattern to broaden even more in the vertical, while the secondary maxima in the horizontal direction have grown to approximately $1 / 2$ of the main central maxima. This last figure clearly illustrates how poor matching of the source to the microscope would lead to a substantial reduction in spatial resolution. However, it appears that a reasonable compromise can be attained whereby the long wavelengths are allowed to overfill the objective while the short wavelengths underfill slightly (as in the middle panel).

We mention in passing that other objective accessories utilize even more restricted portions of the aperture, leading to complex diffraction-limited spot patterns at the specimen. The attenuated total reflection (ATR) and grazing incidence objectives are examples.

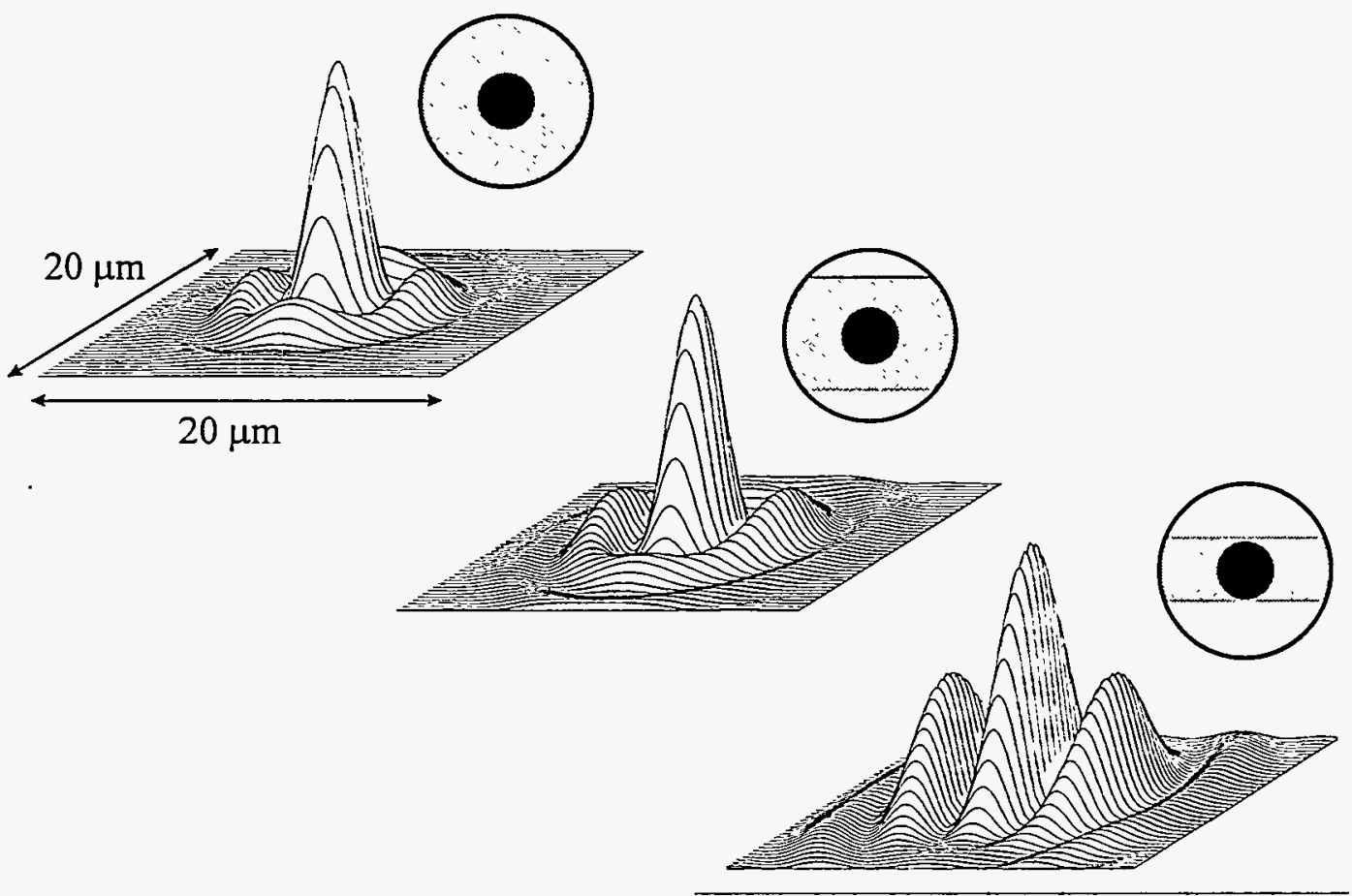

Figure 5. Huygen's point spread function (PSF) at the specimen location (focus) for three difference illumination conditions at the entrance pupil of a $32 \mathrm{X}$ Schwarzchild objective. The diagram adjacent to each PSF graph illustrates the entrance pupil (and secondary obscuration) with the particular illumination pattern shown by gray shading. 
Before addressing some detector issues, we describe one last optical effect that occurs when working at very small spotsizes. Some measurements involve the use of a substrate or windows, made of materials such as $\mathrm{KBr}$ and $\mathrm{BaF}_{2}$, to either support or compress a specimen. Most of these materials have some degree of dispersion in the visible, infrared, or both. For typical thicknesses of these materials ( 1 to $2 \mathrm{~mm}$ ), this dispersion leads to focusing errors of $20 \mu \mathrm{m}$ or more, and severe loss of signal or spatial resolution occurs over part, or all, of the spectral range of interest. A more detailed analysis and discussion will be presented in a subsequent paper ${ }^{6}$.

\section{Detectors}

The standard detector for mid-infrared microspectroscopy is photoconductive mercury cadmium telluride $\left(\mathrm{Hg}_{1-x} \mathrm{Cd}_{\mathrm{x}} \mathrm{Te}\right.$ or MCT$)$ at $\mathrm{T}=77 \mathrm{~K}$. The long wavelength cutoff for this tunable alloy system is usually set to $\sim 16 \mu \mathrm{m}$ $\left(650 \mathrm{~cm}^{-1}\right)$, which provides a good compromise between spectral range and low-noise. Longer wavelength MCT detectors (e.g. to $22 \mu \mathrm{m}$ or $450 \mathrm{~cm}^{-1}$ ) are also available, but performance is reduced by a factor of 2 to 5 compared to $650 \mathrm{~cm}^{-1}$ detectors. Since detector noise increases with area, the detector element is chosen as small as possible. Sizes of $100 \mu \mathrm{m}$ to $250 \mu \mathrm{m}$ are typical for this application. One drawback of the MCT detector is its non-linear response. Electronic circuits to compensate for this are sometimes employed when photometric accuracy is needed, but this may not be enough when performing microspectroscopy with a high brightness source, leading to a highly localized intensity on the detector.

We have overcome some of MCTs limitations by using an extrinsic germanium photoconductive detector with Winston light cone optics. When doped with copper (i.e. $\mathrm{Ge}: \mathrm{Cu}$ ), the long wavelength response extends out to $30 \mu \mathrm{m}$. One drawback is the need for operation at $\mathrm{T}=4.2 \mathrm{~K}$ (liquid helium) as well as a somewhat poorer $\mathrm{D}^{*}$. The Winston cone limits the detector's field of view in order to reduce background noise. But unlike a simple cooled light baffle, the cone behaves like a lens system with a focal plane at its entrance such that a cooled aperture stop can be used to further limit the background radiation reaching the detector. Light exits from the rear of the cone and fills a small cavity where the detector element is situated, providing more homogeneous illumination and minimizing non-linear behavior.

We have adapted a Spectra-Tech Ir $\mu$ s microspectrometer system to accommodate an external detector system. This was achieved by placing a small plane mirror in the optical train before the detector optics and bringing collimated light out through an aperture cut into the side of the spectrometer's case. An off-axis paraboloid $(6.4 \mathrm{~cm}$ effective focal length) is used to focus this beam onto the entrance aperture of the Ge:Cu detector's light cone. The small plane mirror is mounted on a sliding rail so that it can be easily moved in and out of position, thus allowing the optical path to be switched between the internal and external detectors. The (amplified) MCT detector signals were identified in the ribbon cable that connects the MCT to the spectrometer electronics, and brought to a BNC connector and switch mounted onto the back of the spectrometer case. The switch allows one to choose between the internal (MCT) detector signal and the signal from an external detector, fed into the BNC connector. The Ge:Cu detector is equipped with its own pre-amplifier, with gain set manually.

To improve the $\mathrm{Ge}: \mathrm{Cu}$ detector's performance, a $500 \mu \mathrm{m}$ diameter cooled aperture was placed at the entrance of the light cone. This aperture does not restrict the microspectrometer's throughput even for $100 \mu \mathrm{m}$ sampling areas, but substantially reduces the background radiation reaching the detector, as indicated by the increase in detector resistance to above $10 \mathrm{M} \Omega$. In this situation, amplifier noise is likely to dominate (due to the "warm" feedback resistor used in the first-stage op-amp) such that a further reduction in aperture is not expected to enhance performance without modification to the electronics.

In Figure 6 we show a signal-to-noise comparison between the two detectors. The MCT delivers about twice the $\mathrm{S} / \mathrm{N}$, but cuts off at $650 \mathrm{~cm}^{-1}$. The Ge:Cu performs well to $400 \mathrm{~cm}^{-1}$, at which point the FTIR's $\mathrm{KBr}$ beamsplitter becomes opaque. In Figure 7 we show measurements of a $10 \%( \pm 1 \%)$ infrared neutral density filter made with each detector. The results with the $\mathrm{Ge}: \mathrm{Cu}$ agree with the filter's specifications, whereas the MCT results are in error by more than $30 \%$ ! We note that the high brightness of the synchrotron source may aggravate the poor linearity of MCT. In any event, these results indicate that one should proceed with caution when using an MCT detector for measurements requiring good quantitative accuracy. 


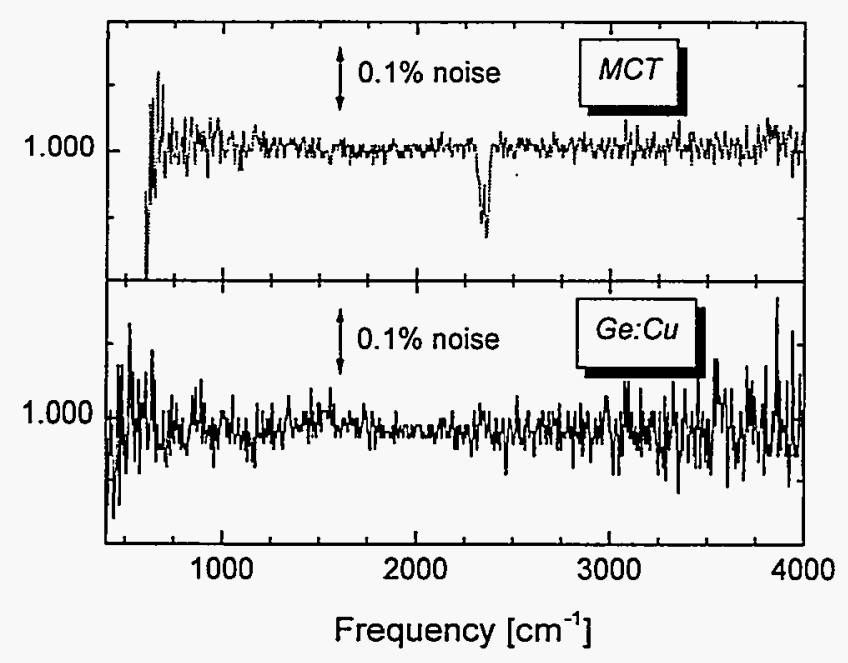

Figure 6. Ratio of two measurements with no sample (100\% lines) that illustrate the available signal to noise for both detector systems.

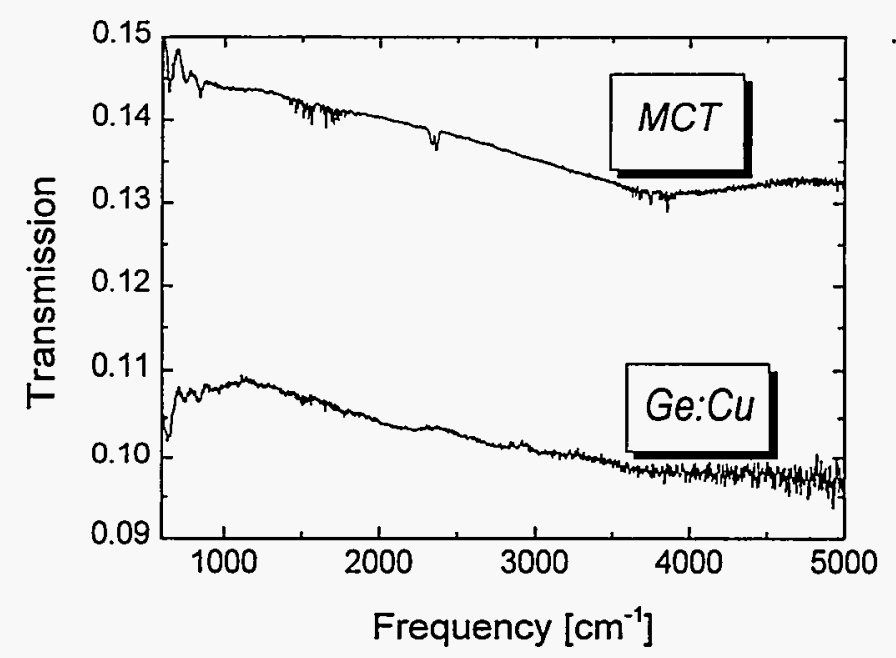

Figure 7. Measured transmission of a $10 \%( \pm 1 \%)$ neutral density filter (Oriel model) for MCT and Ge:Cu detectors. The non-linear performance of the MCT is clearly evident.

\section{Summary}

We have considered the detailed optical system for adapting high brightness synchrotron infrared to a microspectrometer system. When carefully positioned, spherical mirrors can be used to focus and collimate the synchrotron radiation while maintaining essentially all of the intrinsic source brightness. The synchrotron source brightness can be sufficient to allow the use of apertures (field stops) that are effectively smaller than the diffraction limit. In this situation, the spatial resolution achieved by the instrument varies with both wavelength and the effective aperture of the microscope's objectives. Under-filling of the objective must be avoided to ensure the best possible spatial resolution. Also, the diffraction limited resolution for a reflectance measurement is somewhat poorer than for a transmission measurement. Lastly, alternatives to the standard MCT IR detector are available and can be used with the microscope to improve linearity and extend the wavelength range with a modest reduction in signal-to-noise.

\section{Acknowledgments}

We gratefully acknowledge important discussions with R.P. Madden, T. Lucatorto, U. Arp, and A. Hight Walker (NIST), J.A. Reffner (Spectra-Tech, Inc.) and L.M. Miller (Albert Einstein College of Medicine). Special thanks go to the Northrop Grumman Corporation for providing the Ir $\mu$ s instrument. This work was conducted as part of a Cooperative Research and Development Agreement between the Northrop Grumman Corporation and Brookhaven National Laboratory. The National Synchrotron Light Source and BNL are operated for the U.S. Department of Energy under contract DE-AC02-76CH00016.

\section{References}

1. G.P. Williams, Nucl. Instr. Methods 195383 (1982); W.D. Duncan and G.P. Williams, Applied Optics 222914 (1983).

2. G.L. Carr, M. Hanfland and G.P. Williams, Rev. Sci. Instr. 661643 (1995).

3. J.A. Reffner, P.A. Martoglio and G.P. Williams, Rev. Sci. Instr. 661298 (1995); G.L. Carr, J.A. Reffner and G.P. Williams, Rev. Sci. Instr. 661490 (1995).

4. G.L. Carr, D. DiMarzio and D.J. Larson, Jr., Semiconductor Characterization, Present Status and Future Needs, W.M. Bullis, D.G. Seiler and A.C. Diebold, editors, AIP Press p418 (1995).

5. Zemax software for Windows/PC, Focus Software, Inc.

6. L.M. Miller and G.L. Carr, in preparation. 